\title{
An Unusual Presentation of a Thyroid Malignancy: A Case Report
}

\author{
Irfan $\mathrm{M}^{\mathrm{a}}$, Baharudin $\mathrm{A}^{\mathrm{a}}$ and Ananda Dorai $\mathrm{A}^{\mathrm{b}}$ \\ aDepartment of Otorhinolaryngology-Head \& Neck Surgery, ${ }^{b}$ Reconstructive Sciences Unit, School of Medical \\ Sciences, Universiti Sains Malaysia Health Campus, 16150 Kota Bharu, Kelantan
}

\begin{abstract}
Patient with thyroid mass rarely presents in emergency situation. It is because the nature of the mass is that it usually increases slowly in size. Thus, any surgical intervention can be planned electively. The most common condition that necessitates urgent intervention, is upper airway compromise due to sudden rapid increase in size of the swelling. This can be due to intralesional bleeding or superimposed infection on the enlarged gland. We report a case of urgent completion thyroidectomy performed to a patient with fungating recurrent papillary carcinoma who present with bleeding from the mass externally.
\end{abstract}

KEYWORDS: Bleeding, Recurrent papillary carcinoma, Thyroidectomy

\section{CASE REPORT}

A 69 year-old Malay female was diagnosed to have papillary thyroid carcinoma. She had undergone total thyroidectomy few years ago and was scheduled for radioiodine ablation treatment. However she defaulted the appointment.

She was apparently well until 3 months before presentation to the oncology unit whereby she started to notice a mass arising from the previous scar. The mass rapidly increased in size. In addition, the mass also started bleeding. This require her to change the dressing twice a day. The dressings were always soaked with blood.

She was admitted few times to other hospitals and required blood transfusions due to the declining hemoglobin level. She was referred to the oncology team for palliative radiotherapy and subsequently referred to otorhinolaryngology team for possibility of debulking the mass.

In view of poor prognosis of radioiodine uptake with such a big mass, together with the current bleeding problem, urgent completion thyroidectomy was planned. Computed tomography scan was done that showed the mass had invaded the strap muscles and displaced the sternomastoid and great vessels poste-

Corresponding author;

Dr Irfan Mohamad

Department of Otorhinolaryngology-Head \& Neck

Surgery

School of Medical Sciences

Universiti Sains Malaysia Health Campus

e-mail: irfan@kb.usm.my rolaterally.

The patient was stabilized medically and taken to the operation theatre. An elliptical incision with $2 \mathrm{~cm}$ safe margin from the mass on the skin was carried out. Strap muscles were identified and the origin of the mass was traced up to the trachea. The anterior part of the trachea was found to be fibrosed and scarred. The remnant of Berry ligament was curetted and removed together with the mass.

The skin defect was $13 \times 5.5 \mathrm{~cm}$. The closing of the skin was made with the use of pedicled islanded pectoralis major myocutaneous flap and deltopectoral fasciocutaneous flap. Post operative period was uneventful.

\section{DISCUSSION}

Surgical management of thyroid lesion usually will be carried out in an elective manner. It is due to the nature of the disease that progresses slowly. Rarely, it needs urgent or emergency surgical procedure because of the nature if the disease.

Emergency thyroidectomy is usually performed because of upper airway obstruction. The sudden increase in the thyroid swelling that can cause upper airway obstruction include intralesional parenchymal hemorrhage and superimposed infection. ${ }^{1,2}$ In most of the instances the operation can be performed under general anaesthesia. However, in severe upper airway obstruction, regional anaesthesia may be required. ${ }^{3}$

Urgent or emergency thyroidectomy is rarely advocated for a bleeding thyroid mass. It is because many of the superficial bleed will stop with conservative measures such as compression bandage. When the active bleeding controlled, an elective surgery will be performed.

Our case demonstrated that the conservative mea-

\section{.}

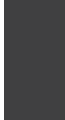


sures failed to halt the bleeding. Most probably it was because the origin was from a malignant tumour compared to the bleeding caused by superficial ulceration or infection. The operation has to be performed urgently because the patient was anaemic and require blood transfusion to maintain her hemoglobin level.

Performing surgery in this patient carries a higher risk of complications. Since this was a revision surgery, the mass already bled preoperatively, she's an elderly patient and the mass has already fungated externally. Removal of skin with safe margin is required and primary reconstruction should be planned. Revision surgery carries a higher risk of recurrent laryngeal nerve injury in view of adhesion and scarring from the first surgery. The rate of laryngeal injury was higher in patients over 65 year old. ${ }^{4}$

The surgery was carried out smoothly. Bleeding was noted only from the tumour bulk and after removal; it stopped with minimal electrical cauterization on the remnant tissue. Skin closure need to be planned earlier. This was due to the direct involvement of the skin by the lesion and also there is a need to remove part of the normal adjacent skin to obtain the safe surgical margin. In our case, pedicled islanded pectoralis major myocutaneous flap was used. Other techniques in similar cases reported were rotational adjacent skin flap and deltopectoral flap. ${ }^{5}$

Post-operative period was uneventful. Drains were kept only for few days. Recurrent laryngeal functions were intact. The patient was discharged with appointment for radioiodine ablation.

\section{CONCLUSION}

Although the patient had undergone total thyroidectomy previously, the tumour recurrence was expected because she default the post-operative radioiodine ablation treatment. However, the usual presentation of recurrent cases will be a midline swelling of the neck. Rarely, it presents with a bleeding fungating mass. Surgical management was the primary treatment. There was a need to carry it out urgently because of the continuous bleeding that did not stop with conservative compression bandage. Post-operatively, the patient was referred back to the oncology for radioiodine ablation treatment.

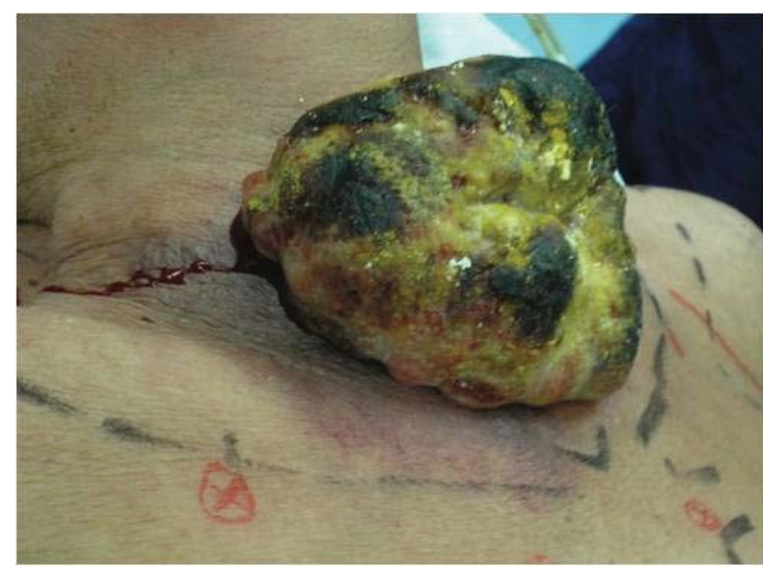

Figure1. Fungating mass from the previous thyroid scar

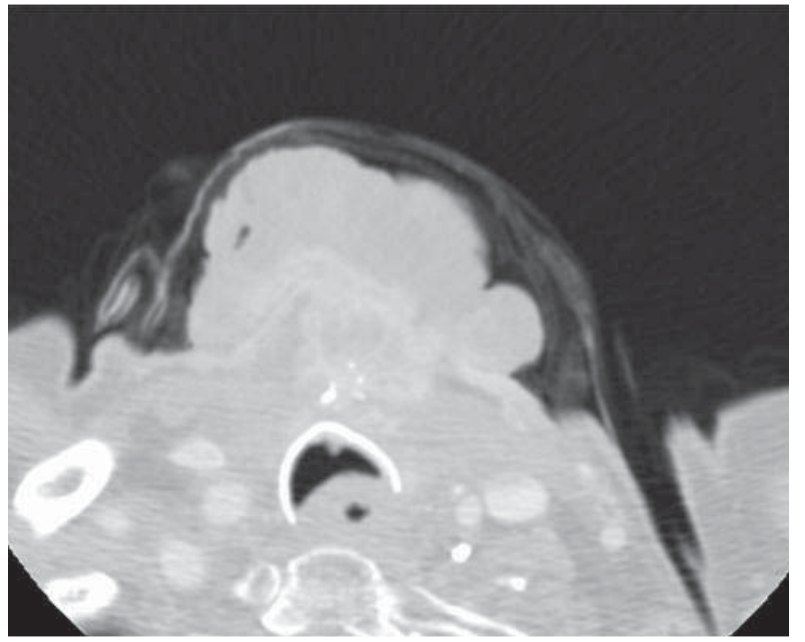

Figure 2. The mass originated from anterior tracheal wall invading the strap muscles

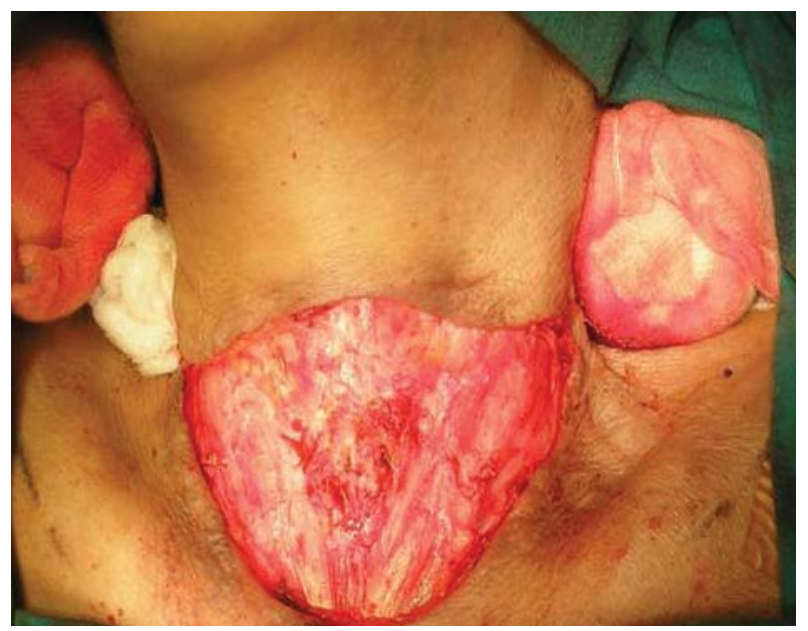

Figure 3. The mass was removed leaving the fibrotic area of anterior tracheal wall.

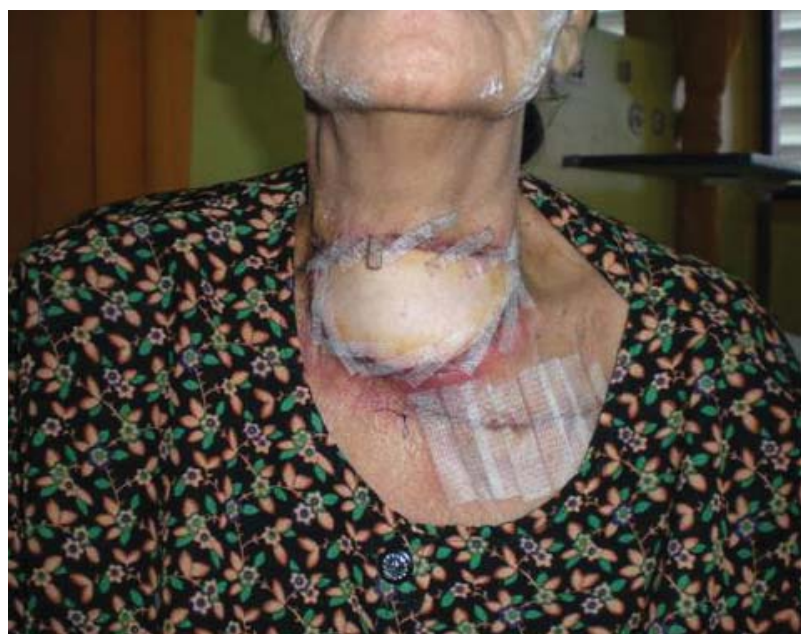

Figure 4. The skin defect was closed with regional pedicled flap. 


\section{REFERENCES}

1. Testini M, Gurrado A, Lissidini G, et al. Emergency surgery for acute respiratory failure secondary to spontaneous thyroid hemorrhage. Int Surg 2008; 93:158-62

2. Masood R, Samiullah, Chaudhary IA, et al. Emergency thyroidectomy in infected thyroid cyst due to spontaneous gas forming organisms. J Coll Physicians Surg Pak 2008; 18:716-8

3. Kalowale IK, Rahman GA. Emergency thyroidectomy in a patient with severe upper airway obstruction caused by goiter: Case for regional anaesthesia. J Natl Med Assoc 2006; 98:86-9

4. Echternach M, Maurer C, Mencke T, et al. Laryngeal complications after thyroidectomy: Is it always the surgeon? Arch Surg 2009; 144:149-53

5. Harjit KD, Hisham AN. Large fungating thyroid cancers: A unique surgical challenge. Asian J Surg 2005; 28:48-51 
IMI THE INTERNATIONAL MEDICAL JOURNAL

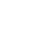
(1) 